\title{
TITLE:
}

\section{ON THE DEFORMATION OF A CERTAIN TYPE OF ALGEBRAIC VARIETIES}

AUTHOR(S):

OKA, Mutsuo

\section{CITATION:}

OKA, Mutsuo. ON THE DEFORMATION OF A CERTAIN TYPE OF ALGEBRAIC VARIETIES. 数理 解析研究所講究録 1987, 634: 72-92

ISSUE DATE:

1987-12

URL:

http://hdl.handle.net/2433/100089

RIGHT: 
ON THE DEFORMATION OF A CERTAIN TYPE OF ALGEBRAIC VARIETIES

Mutsuo OKA

Dedicated to Professor I. Tamura for his $60^{\text {th }}$ birthday

\section{\$1. Introduction}

Let $A=\left(a_{i j}\right)(1 \leqq i, j \leqq n)$ be an upper triangular integral matrix with a non-zero determinant and $a_{i j} \geqq 0$ for each $i, j$. Let $\Delta$ be the $n$-simplex in $\mathbf{R}^{n}$ which is spun by $A_{0}=\vec{D}$ and $A_{i}=\left(a_{i 1}, \ldots, a_{i n}\right)(i=1, \ldots, n)$. Let $A_{n+1}$, $A_{n+2}, \ldots, A_{l}$ be the other integral points in $\Delta$. For an integral vector $\nu=\left(\nu_{1}, \ldots, \nu_{n}\right)$, we denote the monomial $y_{1}^{\nu_{1}} \ldots y_{n}^{\nu}{ }^{\nu}$ by $y^{\nu}$. For $t=\left(t_{0}, \ldots, t_{l}\right)$ of $c^{l+1}$, we definé

$$
h(y, t)=t_{0}+\sum_{j=1}^{\ell} t_{j} y^{A} j
$$

and let $M_{t}^{a}$ be the affine variety in $c^{n}$ defined by $h(y, t)=0$. There exists a toric variety $w$ of dimension $n$ which depends only on $\Delta$ and a Zariski open subset $U$ of $c^{l+1}$ such that $w \supset \mathbf{C}^{n} \supset M_{t}^{a}$ and the closure $M_{t}$ of $M_{t}^{a}$ in $W$ is nonsingular for each $\mathbf{t} \in \mathbf{U}$. This type of algebraic variety $\mathbf{M}_{\mathbf{t}}$ appears as an exceptional divisor of a resolution of an 
isolated hypersurface singularity ([12]). The purpose of this paper is to study this deformation $\left\{M_{t}\right\}$ in $W$.

In $\$ 5$, we prove the surjectivity of the infinitesimal displacement map

$$
\xi: \mathrm{T}_{\mathbf{t}} \mathrm{U} \rightarrow \mathrm{H}^{0}\left(\mathrm{M}_{\mathbf{t}}, \nu_{\mathbf{t}}\right) \text {. }
$$

In $\$ 6$, we give a criterion about the injectivity of the Kodaira-Spencer map

$$
\delta \cdot \xi^{e}: \mathrm{T}_{\mathbf{t}} \mathrm{U}^{\mathrm{e}} \longrightarrow \mathrm{H}^{1}\left(\mathrm{M}_{\mathbf{t}},{ }_{\mathrm{t}}\right)
$$

In $\S 7$, we will apply the results in $\$ \S 5,6$ to construct a complete deformation of a Godeaux surface.

\section{$\S 2$. Infinitesimal displacement}

Let $W$ be a compact complex manifold of dimension $n$ and let $\left\{M_{t}\right\}(t \in U)$ be an analytic family of non-singular hypersurfaces where $U$ is an open set of $c^{\ell+1}$. Let $\left\{\left(U_{\alpha}, z_{\alpha}\right)\right\} \quad(\alpha \in S)$ be local coordinate systems of $W$ such that (i) $W=\underset{\alpha \in S}{U} U_{\alpha}$ and ( $i$ ) there exists analytic functions $f_{\alpha}\left(z_{\alpha}, t\right)$ on $U_{\alpha} x U$ such that $M_{t} \cap U_{\alpha}=\left\{z_{\alpha} \in U_{\alpha} ; f_{\alpha}\left(z_{\alpha}, t\right)=0\right\}$. Let $h_{\alpha \beta}=f_{\alpha} / f_{\beta}$. We may assume that $h_{\alpha \beta} \in \theta^{*}\left(U_{\alpha} \cap_{\beta}\right)$. The line bundle $\left[\mathrm{M}_{\mathrm{t}}\right]$ is defined by the cocycle $\left\{\mathrm{h}_{\alpha \beta}\right\}$ of $\mathrm{H}^{1}\left(\mathrm{~W}, Q^{*}\right)$ and the normal bundle $N_{t}$ of $M_{t}$ in $W$ is the restriction of $\left[M_{t}\right]$ to $M_{t}$. Let $\nu_{t}$ be the sheaf of the germs of the holomorphic sections of $N_{t}$. Take a holomorphic tangent vector $v \in T_{t} U$. As $\mathrm{f}_{\alpha}=\mathrm{h}_{\alpha \beta} \mathrm{f}_{\beta}$, we have

$$
v\left(f_{\alpha}\right)=h_{\alpha \beta} v\left(f_{\beta}\right) \text { on } U_{\alpha} \cap U_{\beta} \cap M_{t} \text {. }
$$


This defines a canonical linear mapping

$$
\xi: \mathrm{T}_{\mathbf{t}} \mathrm{U} \rightarrow \mathrm{H}^{0}\left(\mathrm{M}_{\mathbf{t}}, \nu_{\mathbf{t}}\right)
$$

where $\xi(v)=\left\{v\left(f_{\alpha}\right)\right\}(\alpha \in S) . \quad \xi(v)$ is called the infinitesimal displacement along $\underline{v}$.

Let $\theta_{W}$ and $\theta_{t}$ be the sheaves of the germs of holomorphic vector fields of $W$ and $M_{t}$ respectively. We have the exact sequence of sheaves:

$$
0 \rightarrow \Theta_{\mathbf{t}} \rightarrow \Theta_{\mathbf{W}} \mid \mathrm{M}_{\mathbf{t}} \rightarrow \nu_{\mathbf{t}} \rightarrow 0
$$

This induces the following exact sequence.

$$
\begin{aligned}
& 0 \rightarrow H^{0}\left(M_{t}, \theta_{t}\right) \rightarrow H^{0}\left(M_{t},{ }{ }_{W} \mid M_{t}\right) \rightarrow H^{0}\left(M_{t}, \nu_{t}\right) \\
& \stackrel{\delta}{\longrightarrow} H^{1}\left(M_{t}, \theta_{t}\right) \longrightarrow H^{1}\left(M_{t},{ }^{\theta_{W}} \mid M_{t}\right) \longrightarrow \cdots
\end{aligned}
$$

The composition

$$
\mathrm{T}_{\mathbf{t}} \mathrm{U} \stackrel{\xi}{\longrightarrow} \mathrm{H}^{0}\left(\mathrm{M}_{\mathbf{t}}, \nu_{\mathbf{t}}\right) \stackrel{\delta}{\longrightarrow} \mathrm{H}^{1}\left(\mathrm{M}_{\mathbf{t}}, \theta_{\mathbf{t}}\right)
$$

is equal to the infinitesimal deformation map. See Kodaira-Spencer [6] or Kodaira [7] for details.

\section{§3. Resolution of a hypersurface singularity}

We recall basic properties about the resolution of a hypersurface singularity through the toroidal embedding theory. We use the same notation as in [12]. Let $f\left(z_{0}, \ldots, z_{n}\right)=\sum_{\nu} a_{\nu} z^{\nu}$ be an analytic function defined in a 
neighborhood of the origin and we assume that $\mathrm{V}=\mathrm{f}^{-1}(0)$ has an isolated singular point at the origin. Let $\Gamma_{+}(f)$ be the convex hull of $U_{\nu} \neq 0 \leq\left\{\nu+\left(\mathbf{R}^{+}\right)^{n+1}\right\}$. The Newton boundary $\Gamma(f)$ is the union of the compact faces of $\Gamma_{+}(f)$. We assume that $f$ is non-degenerate on each face $\Delta$ of $\Gamma(f)$. Let $N$ be the dual space Hom $\left(\mathbf{R}^{\mathrm{n}+1}, \mathbf{R}\right)$. We identify $N$ with $\mathbf{R}^{\mathrm{n}+1}$ through the standard inner product and we denote the dual vectors by column vectors to avoid confusion. Let $\mathrm{N}^{+}$be the set of non-negative dual vectors. We introduce an equivalence relation $\sim$ in $\mathrm{N}^{+}$by $P \sim Q$ if and only if $\Delta(P)=\Delta(Q)$. Here $\Delta(P)$ is the locus where the restriction of $\mathrm{P}$ on $\Gamma_{+}(f)$ takes its minimal value which we denote by $d(P)$. This induces a cone-like polyhedral decomposition of $\mathrm{N}^{+}$and we denote this by $\Gamma^{*}(f)$. Let $\Sigma^{*}$ be a unimodular simplicial subdivision. For each n-simplex $\sigma=\left(P_{0}, \ldots, P_{n}\right)=\left(p_{i j}\right)$ which is a unimodular matrix, we associate an affine space $c_{\sigma}^{n+1}$ with coordinate $\mathbf{y}_{\sigma}=\left(y_{\sigma 0}, \ldots, y_{\sigma n}\right)$. Let $\pi_{\sigma}: \mathbf{c}_{\sigma}^{n+1} \rightarrow \mathbf{c}^{n+1}$ be the birational morphism defined by $\pi\left(y_{\sigma}\right)=\left(z_{0}, \ldots, z_{n}\right)$ where $z_{i}=\prod_{j=0}^{n} y_{\sigma j}^{p_{i j}}$. Let $x$ be the complex manifold of dimension $n+1$ which is obtained by gluing the affine spaces $c_{\sigma}^{n+1}$ where $\sigma$ moves in the $n$-simplices of $\Sigma^{*}$ and let $\hat{\pi}: x \rightarrow c^{n+1}$ be the projection map. Let $\widetilde{V}$ be the proper transform of $V$ and let $\pi: \widetilde{V} \rightarrow V$ be the restriction of $\hat{\pi}$ to $\tilde{V}$. By the nondegeneracy assumption, $\pi: \widetilde{V} \rightarrow V$ is a good resolution of $V$. For each strictly positive vertex $P$ of $\Sigma^{*}$ with $\operatorname{dim} \Delta(P) \geq 1$, there are corresponding exceptional divisors $\hat{E}(P)$ and $E(P)$ 
of $\hat{\pi}$ and $\pi$ respectively so that $E(P)$ is a hypersurface in $\hat{E}(P)$. $\hat{E}(P)$ is a toric variety. Let $\sigma=\left(P_{0}, \ldots, P_{n}\right)$ with $P$ $=P_{0}$. Then in the coordinate chart $c_{\sigma}^{n+1}, \hat{E}(P)$ is defined by $y_{\sigma 0}=0$ and $E(P)$ is defined by $\hat{E}(P) \cap\left\{h_{\sigma}\left(y_{\sigma 1}, \ldots, \dot{y}_{\sigma n}\right)=0\right\}$ where $h_{\sigma}\left(y_{\sigma}\right)$ is defined by

(3.1) $\quad f_{\Delta(P)}\left(\pi_{\sigma}\left(y_{\sigma}\right)\right)=\prod_{i=0}^{n} y_{\sigma i}^{d\left(P_{i}\right)} h_{\sigma}\left(y_{\sigma 1}, \ldots, y_{\sigma n}\right)$.

$\S 4$. Compactification of $M_{t}^{a}$.

Let $h(y, t)$ be as in (1.1). Let $\sigma^{\prime}$ be the unimodular

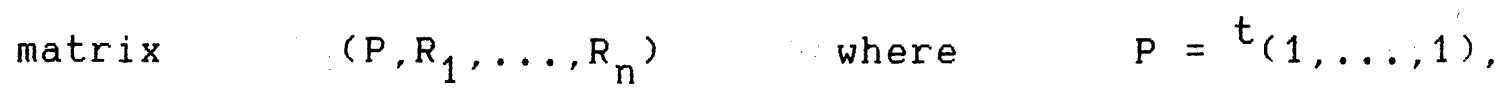
$R_{1}=t(0,1, \ldots, 0)$, Let $\pi_{\sigma^{\prime}}: c^{n+1} \rightarrow c^{n+1}$ be as in $\S 3$. Let $y_{0}, \ldots, y_{n}$ be the coordinate of the source. Then we have $z_{0}=y_{0}$ and $z_{i}=y_{0} y_{i}$ for $i=1, \ldots, n$. Let $k$ be the degree of $h$ and we define $f_{\Xi}(z, t)=h\left(\pi_{\sigma}^{-1}(z, t)\right) z_{0}^{k}=$ $h\left(z_{1} / z_{0}, \ldots, z_{n} / z_{0}, t\right) z_{0}^{k}$. Then $f_{\Xi}(z, t)$ is a homogeneous polynomial in $z_{0}, \ldots, z_{n}$ and we can write

$$
f_{\Xi}(z, t)=\sum_{i=0}^{\ell} t_{j} z^{B}
$$

for some integral vectors $B_{0}, \ldots, B_{\ell} \cdot$ Note that $B_{0}=(k, 0, \ldots, 0)$. Let $f(z, t)=f_{\Xi}(z, t)+\sum_{i=0}^{n} z_{i}^{L}$ for a sufficiently large L. The notation $f_{\Xi}(z)$ is the same as in [12] if we set $\Xi=\Delta(P)$. There exists a Zariski open subset $U$ of $c^{l+1}$ such that $f(z, t)$ has a non-degenerate Newton boundary for each $t \in U$. Let $\sigma=\left(P, P+R_{1}, \ldots, P+R_{n}\right)$. If $L$ is 
sufficiently large, $\Delta\left(P+P_{i}\right) \supset B_{0}$ for each $i=1, \ldots, n$. Thus $\sigma$ is an admissible simplex of $\Gamma^{*}(f)$. ( $\sigma^{\prime}$ is not necessarily an admissible simplex.) Thus we can take a unimodular simplicial subdivision $\Sigma^{*}$ which has $\sigma$ as an $n-s i m p l e x$ by $\$ 3$ of [12].

Assertion. The defining equation of $E(P)$ in $c_{\sigma}^{n+1} \cap\left\{y_{\sigma 0}=0\right\} \underline{\text { is equal }}$ to $h\left(y_{\sigma}, t\right)=0$.

Proof. E(P) is defined by $h_{\sigma}\left(y_{\sigma}, t\right)=0$ where

$$
\begin{aligned}
h_{\sigma}\left(y_{\sigma}, t\right) & =f_{\Delta}\left(\pi_{\sigma}\left(y_{\sigma}\right), t\right) / y_{\sigma 0}^{d(P)} \prod_{i=1}^{n} d(P)+d\left(R_{i}\right) \\
& =f_{\Delta}\left(\pi_{\sigma},(y)\right) /\left\{\left(y_{\sigma 0} \ldots y_{\sigma n}\right) d(P) \prod_{i=1}^{n} y_{\sigma i} d\left(R_{i}\right)\right\} \\
& =h(y, t)=h\left(y_{\sigma}, t\right)
\end{aligned}
$$

Here we have used the equality $\pi_{\sigma}^{-1} \cdot \pi_{\sigma}=\pi_{\sigma},-1 \sigma$ and $y_{0}=y_{\sigma 0} \ldots y_{\sigma n}$ and $y_{i}=y_{\sigma i}$ for $i=1, \ldots, n$.

Thus we take $E(P)$ as the compactification $M_{t}$ of $M_{t}^{a}$ and $\hat{E}(P)$ as $W$ hereafter. Note that $\pi_{1}\left(M_{t}\right)$ is a finite cyclic group by Theorem (7.3) of [12]. Let $S$ be the set of the n-simplex $\tau$ of $\Sigma^{*}$ such that $P$ is a vertex of $\tau$. Then it is obvious that $\left\{\mathrm{C}_{\sigma}^{\mathrm{n}}\right\} \quad(\sigma \in \mathrm{S})$ is an open covering of $\mathrm{W}$ where $\mathbf{c}_{\sigma}^{\mathrm{n}}=\mathbf{c}_{\sigma}^{\mathrm{n}+1} \cap\left\{\mathrm{y}_{\sigma 0}=0\right\}$.

Remark. To study the deformation of $M_{t}$ in $w$, we only need the information about $s$. 


\section{$\S 5$. Main theorem}

We are ready to state the main theorem. Let $\nu_{t}$ be the sheaf of the germs of the holomorphic sections of the normal bundle $N_{t}$ of $M_{t}$ in $W$. Let $\ell$ be as in $\$ 1$.

Theorem (5.1). (i) $\operatorname{dim} H^{0}\left(M_{t}, \nu_{t}\right)=l$ and the infinitesimal displacement map $\xi: T_{t} U \rightarrow H^{0}\left(M_{t}, \nu_{t}\right)$ is suriective. The kernel of $\xi$ is generated by $\sum_{j=0}^{l} t_{j} \frac{\partial}{\partial t_{j}}$.

(ii) Let $\psi_{1}, \ldots, \psi_{\ell}$ be a system of the generators of $H^{0}\left(M_{t}, \nu_{t}\right)$ and let $\Psi: M_{t} \rightarrow P^{l-1}$ be the associated mapping. Then $\Psi$ is a birational morphism.

Let $W=\hat{E}(P)$ and $M_{t}=E(P)$ as in $\S 4$. For each $n$-simplex $\tau=\left(Q_{0}(\tau), \ldots, Q_{n}(\tau)\right)$ of $S$, we may assume that

$$
Q_{0}(\tau)=P \text {. }
$$

Let $h_{\tau}\left(y_{\tau}, t\right)$ be the defining polynomial of $M_{t}$ in $c_{\tau}^{n}=c_{\tau}^{n+1} \cap\left\{y_{\tau 0}=0\right\} . h_{\tau}$ is defined by the equality

$$
f_{\Delta}\left(\pi_{\tau}\left(y_{\tau}\right), t\right)=\prod_{i=0}^{n} y_{\tau i}^{d\left(Q_{i}(\tau)\right)} h_{\tau}\left(y_{\tau}, t\right) .
$$

Take two simplices $\alpha$ and $\beta$ in $s$ and let $\alpha^{-1} \beta=\left(\lambda_{i j}\right)$ $(0 \leqq i, j \leqq n)$. By $(5.2)$, we have $\lambda_{00}=1$ and $\lambda_{i 0}=0$ for $i$ $=1, \ldots, n$. Recall that $\mathbf{c}_{\alpha}^{\mathrm{n}}$ and $\mathrm{c}_{\beta}^{\mathrm{n}}$ are glued by

$$
y_{\alpha i}=\prod_{j=1}^{n} y_{B j}^{\lambda} \quad(i=1, \ldots, n)
$$


Now we consider the line bundle $\left[M_{\mathbf{t}}\right]$ which is defined by the cocycle $\left\{h_{\alpha \beta}\right\}$ where $h_{\alpha \beta}=h_{\alpha} / h_{\beta} \cdot$ By (5.3), we have (5.5) $\quad h_{\alpha \beta}\left(y_{\beta}, t\right)=\prod_{i=0}^{n} y_{\beta i}^{d\left(Q_{i}(\beta)\right)}, \prod_{i=0}^{n} y_{\alpha i} d\left(Q_{i}(\alpha)\right)$.

Here the right hand is considered as a monomial of $y_{\beta 1}, \ldots$. $\mathbf{y}_{\beta n}$ through (5.4). The exponent of $y_{\beta 0}$ is zero. We can write $h_{\tau}\left(y_{\tau}, t\right)$ more explicitly as

$$
h_{\tau}\left(y_{\tau}, t\right)=\sum_{j=0}^{\ell} t_{j} y_{\tau}^{A_{j}(\tau)}
$$

where the positive integral vector $A_{j}(\tau)$ is characterized by

$$
\pi_{\tau}\left(y_{\tau}\right)^{B}=\left(\prod_{i=0}^{n} y_{\tau i} d\left(Q_{i}(\tau)\right), y_{\tau}^{A_{j}(\tau)} .\right.
$$

Combining (5.7) and (5.5), we obtain

$$
\mathbf{y}_{\alpha}^{A_{j}(\alpha)}=h_{\alpha \beta} \mathbf{y}_{\beta}^{A_{j}^{(\beta)}} \text {. }
$$

(5.8) says that $\left\{y_{\alpha}{ }^{(\alpha)}\right\}(\alpha \in S)$ is an element of $H^{0}\left(W, \theta\left(\left[M_{t}\right]\right)\right)$. Thus we get the inequality $\operatorname{dim} H^{0}\left(W, \theta\left(\left[M_{t}\right]\right)\right) \geqq \ell+1$. On the other hand, take a monomial $y_{\sigma}^{\mu}$ where $\mu \neq A_{j}(\sigma)$ for $j=0, \ldots, l$. (Here $\sigma$ is fixed.) Let $\Pi_{k}$ be the hyperplane which contains $\left\{A_{i}(\sigma) ; i \neq k, 0 \leqq i \leqq n\right\}$. Then there is an integer $k$ $(0 \leqq k \leqq n)$ such that $A_{k}(\sigma)$ and $\mu$ are separated by $\Pi_{k}$. Take a simplex $\beta=\left(P, Q_{1}(\beta), \ldots, Q_{n}(\beta)\right)$ such that

(5.9) $\quad B_{i} \in \Delta\left(Q_{1}(\beta)\right)$ for $i \neq k, i=0, \ldots, n$. 
Assume that $y_{\sigma}^{\mu}=h_{\sigma \beta} y_{\beta}^{\nu}$ for $\nu=\left(\nu_{1}, \ldots, \nu_{n}\right)$. Then by the assumption, we have $\nu_{1}<0$. This implies that the section $y_{\sigma}^{\mu}$ of $H^{0}\left(C_{\sigma}^{n},\left(\left[M_{t}\right]\right)\right)$ cannot be holomorphically extended to $W$. Thus using GAGA-principle [13], we have proved the following .

Lemma (5.10). $\quad \operatorname{dim} H^{0}\left(w, \theta\left(\left[M_{t}\right]\right)\right)=\ell+1$

and $\left\{y_{\alpha}^{A}{ }^{(\alpha)}\right\}(\alpha \in S),(j=0, \ldots, \ell)$ qives a canonical basis.

This is a special case of $\$ 6$ of [1] and Lemma 2.3 of [10]. For the further geometry of the toric variety $w$, see $[5,2,1,9,3]$.

We are ready to prove (i) of Theorem (5.1). From the exact sequence of sheaves on $W$ :

$$
0 \longrightarrow \theta \longrightarrow \theta\left(\left[M_{t}\right]\right) \longrightarrow v_{t} \longrightarrow 0 .
$$

we have the exact sequence

$$
(5.11) \quad 0 \rightarrow \mathrm{C} \longrightarrow \mathrm{H}^{0}\left(\mathrm{w}, \theta\left(\left[\mathrm{M}_{\mathbf{t}}\right]\right)\right) \stackrel{\theta}{\longrightarrow} \mathrm{H}^{0}\left(\mathrm{M}_{\mathbf{t}}, \nu_{\mathbf{t}}\right) \rightarrow 0
$$

Here we have used the fact that $H^{1}(w)=$,0 because $w$ is simply connected $([1])$. Thus $\operatorname{dim} H^{0}\left(M_{t}, \nu_{t}\right)=\ell$ and $H^{0}\left(M_{t}, \nu_{t}\right)$ is generated by $\varphi_{j}=\left\{y_{\alpha}^{A}{ }^{(\alpha)}\right\}_{\alpha \in S}(j=0, \ldots, l)$. They satisfy the obvious relation $\sum_{j=0}^{\ell} t_{j} \varphi_{j}=0$. Now we study the infinitesimal displacement map $\xi: \mathrm{T}_{\mathbf{t}} \mathrm{U} \rightarrow \mathrm{H}^{0}\left(\mathrm{M}_{\mathbf{t}}, \nu_{\mathbf{t}}\right)$. By the definition of $\xi$, we have

$$
\xi\left(\frac{\partial}{\partial t_{j}}\right)=\left\{\frac{\partial h_{\alpha}}{\partial t_{j}}\right\}_{\alpha \in S}=\left\{y_{\alpha}{ }^{(\alpha)}\right\}_{\alpha \in S}=\varphi_{j} .
$$


Thus $\xi$ is surjective and the kernel of $\xi$ is generated by $\sum_{j=0}^{\ell} t_{j} \frac{\partial}{\partial t_{j}}$. This completes the proof of (i) of Theorem (5.1).

Now we will prove (ii) of Theorem (5.1). Let $\varphi_{0}, \ldots \varphi_{\ell}$ be as above and define $\hat{\Psi}: \mathrm{W} \rightarrow \mathrm{P}^{\ell}$ by $\hat{\Psi}(\mathrm{x})=\left[\varphi_{0}(\mathrm{x}) ; \ldots ; \varphi_{\ell}(\mathrm{x})\right]$. Let $\tau \in S$. As the polynomial $h_{\tau}\left(y_{\tau}\right)$ contains a non-zero constant term, there exists an integer $0 \leqq k \leqq n$ such that $A_{k}(\tau)=(0, \ldots, 0)$. As $\hat{\Psi}\left(y_{\tau}\right)=\left[y_{\tau}^{A_{0}(\tau)} ; \ldots ; y_{\tau}^{A_{l}(\tau)}\right]$ on $c_{\tau}^{n}$, this implies that $\hat{\Psi}$ is a morphism. We have to prove that $\hat{\Psi}$ is generically injective. Note that $\left\{A_{0}(\tau), \ldots, A_{\ell}(\tau)\right\}$ is equal to the set of the integral points of the simplex spun by $A_{j}(\tau)(j=0, \ldots, n)$. By Lemma (3.8) of [12], there exist $0 \leqq i_{1}<\ldots<i_{n} \leqq \ell$ such that $t_{\zeta}=\left({ }^{t} A_{A_{1}}(\tau), \ldots, t_{A_{i}}(\tau)\right)$ is a unimodular matrix. Let $\xi^{-1}=\left(\xi_{i j}\right)$. The image of $\hat{x} \mid c_{\tau}^{n}$ is in the coordinate chart $U_{k}=\left\{X_{k} \neq 0\right\}$ of $P^{l}$. Let $Y_{j}=X_{j} / X_{k}(j \neq k)$. Assume that $\hat{y}\left(y_{\tau}\right)=\left(y_{j}\right)_{j \neq k}$ for $y_{\tau} \in\left(C_{\tau}^{*}\right)^{n}$. Then $y_{\tau}$ is determined by $y_{\tau m}=\prod_{j=1}^{n} Y_{i j}^{\zeta j} \quad(m=1, \ldots, n)$. This proves that $\hat{\Psi}$ is injective on $\left(C_{\tau}^{*}\right)^{n}$. Therefore the restriction of $\hat{\Psi}$ to $M_{t}$ is also a morphism and is injective on $M_{t} \cap\left(C_{\tau}^{*}\right)^{n}$. The image of $\hat{\Psi} \mid M_{t}$ is in the hyperplane $H: \sum_{j=0}^{\ell} t_{j} x_{j}=0$ of $\mathbf{P}^{\ell}$. Identifying $\mathrm{H}$ with $\mathbf{P}^{\ell-1}$, we have $\hat{\Psi} \mid \mathbf{M}_{\mathbf{t}}=\Psi$. This completes the proof of Theorem (5.1).

Remark. If $A=d I_{n}, W$ is the projective space of 
dimension $n$ and $\left\{M_{t}\right\}$ are projective hypersurfaces of degree d. This case is studied in [6].

\section{\$6. Canonical vector fields}

Let $\tau \in S$. Then $\Theta_{W} \mid C_{\tau}^{n}$ is a free -module of rank $n$ with a canonical basis $\left\{\frac{\partial}{\partial y_{\tau 1}}, \ldots, \frac{\partial}{\partial y_{\tau n}}\right\}$. We define $\frac{\tilde{\partial}}{\partial y_{\tau i}}=y_{\tau i} \frac{\partial}{\partial y_{\tau i}}$ for $\quad i=1, \ldots, n$. Similarly we define $\widetilde{d}_{\tau i}=\frac{d y_{\tau i}}{y_{\tau i}}$. Let $\beta \in S$ and let $\beta^{-1} \tau=\left(\lambda_{i j}\right)$ and let $\left(\mu_{i j}\right)=\tau^{-1} \beta$. Then we have

Proposition (6.1). (i) We have the formula

$$
\frac{\tilde{\partial}}{\partial y_{\tau i}}=\sum_{j=1}^{n} \lambda_{j i} \frac{\tilde{\partial}}{\partial y_{\beta j}}, \quad \tilde{d} y_{\tau i}=\sum_{j=1}^{n} \mu_{i j} \tilde{d} y_{\beta j} .
$$

(ii) $\left\{\frac{\tilde{\partial}}{\partial y_{\tau i}} ; i=1, \ldots, n\right\}$ can be holomorphically extended to $\underline{\text { W. }}$

Proof. Recall that $y_{\beta j}=\prod_{i=1}^{n} y_{\tau i}^{\lambda}{ }_{j i}$. Thus the assertion (i) is obvious. The assertion (ii) follows from (i).

Definition (6.2). $\left\{\frac{\tilde{\partial}}{\partial y_{\tau 1}}, \ldots, \frac{\tilde{\partial}}{\partial y_{\tau n}}\right\}$ generates a subspace of dimension $n$ of $\mathrm{H}^{0}\left(\mathrm{~W}, \Theta_{\mathrm{W}}\right)$ which we denote by $\operatorname{Can}\left(W, \theta_{W}\right)$. The restriction of $\operatorname{Can}\left(W, \Theta_{W}\right)$ to $H^{0}\left(M_{t},{ }_{W} \mid M_{t}\right)$ is denoted by $\operatorname{Can}\left(M_{t}, \theta_{W}\right)$. We call vector fields in $\operatorname{Can}\left(W, \theta_{W}\right)$ or in $\operatorname{Can}\left(M_{t}, \theta_{W}\right)$ canonical vector fields. These vector fields come from the torus action on $W$. It is easy to see that 
$\operatorname{dim} \operatorname{Can}\left(M_{t}, \Theta_{W}\right)=n$

Corollary (6.3). We have the inequalities $\operatorname{dim} H^{0}\left(W, \theta_{W}\right) \geqq n$ and $\operatorname{dim} H^{0}\left(M_{t}, \theta_{W} \mid M_{t}\right) \geqq n$.

Now we characterize the image of $\theta: \operatorname{Can}\left(M_{t}, \theta_{W}\right) \rightarrow H^{0}\left(M_{t}, \nu_{t}\right)$. Let $\sigma$ be the fixed simplex so that $h_{\sigma}\left(y_{\sigma}, t\right)=h\left(y_{\sigma}, t\right)$ where $h$ is as in (1.1). Let $x$ $\in H^{0}\left(M_{t},\left.\theta_{W}\right|_{M_{t}}\right)$ and let $x=\sum_{i=1}^{n} x_{\tau i} \frac{\partial}{\partial y_{\tau i}}$ on $c_{\tau}^{n}$. Then it is easy to see that

(6.4) $\theta(X)=\left(\theta(X)_{\tau}\right)_{\tau \in S}$ where $\theta(X)_{\tau}=\sum_{i=1}^{n} x_{\tau i} \frac{\partial h_{\tau}}{\partial y_{\tau i}}$.

Let $x^{1}, \ldots, x^{n}$ be the canonical vector fields defined by

(6.5) $\quad x^{i}=\frac{\tilde{\partial}}{\partial y_{\sigma i}}=y_{\sigma i} \frac{\partial}{\partial y_{\sigma i}}$ on $c_{\sigma}^{n} \quad(i=1, \ldots n)$.

Then we have

$$
\theta\left(x^{i}\right)_{\sigma}=y_{\sigma i} \frac{\partial h}{\partial y_{\sigma i}}(i=1, \ldots n) .
$$

We claim that $\left\{\theta\left(x^{i}\right)\right\}(i=1, \ldots, n)$ are linearly independent. In fact, assume, that $\sum_{i=1}^{n} \lambda_{i} \theta\left(x^{i}\right)=0$. Then we must have $\sum_{j=1}^{\ell} t_{j} b_{j} \mathbf{y}_{\sigma}^{A} \equiv 0$ modulo $h\left(y_{\sigma}, t\right)$ where $b_{j}=\sum_{i=1}^{n} \lambda_{i} a_{j i}$. This implies that $\lambda_{i}=0$ for each $i$. Thus we have shown

Theorem (6.7). $\theta\left(x^{1}\right), \ldots, \theta\left(X^{n}\right)$ are linearly independent. They are characterized by

$$
\theta\left(x^{i}\right)_{\sigma}=\left.\frac{d}{d s} h\left(y_{\sigma 1}, \ldots, s y_{\sigma i}, \ldots, y_{\sigma n}, t\right)\right|_{s=1}
$$


Now we consider the following subfamily of $\left\{M_{t}\right\}$. Let $U^{e}=\left\{t \in U ; t_{0}=\ldots=t_{n}=1\right\}$. We call $\left\{M_{t}\right\}\left(t \in U^{e}\right)$ the embedded deformation. Let $\xi^{e}: \mathrm{T}_{\mathbf{t}} \mathrm{U}^{\mathrm{e}} \rightarrow \mathrm{H}^{0}\left(\mathrm{M}_{\mathbf{t}},{ }^{\nu} \mathbf{t}\right)$ be the restriction of $\xi$ to $\mathrm{T}_{\mathbf{t}} \mathrm{U}^{e}$. Then we have

Theorem (6.8). Assume that $H^{0}\left(M_{t}, \theta_{W} \mid M_{t}\right)=\operatorname{Can}\left(M_{t}, \theta_{W}\right)$. Then the Kodaira-Spencer map $\delta \cdot \xi^{e}: \mathrm{T}_{\mathbf{t}} \mathrm{U}^{\mathrm{e}} \rightarrow \mathrm{H}^{1}\left(\mathrm{M}_{\mathbf{t}},{ }^{\theta} \mathbf{t}\right)$ is iniective and $H^{0}\left(M_{t}, \theta_{t}\right)=0$.

Proof The second assertion is immediate from Theorem $(6.7),(2.4)$ and the assumption. Assume that $\delta \circ \xi^{e}(v)=0$ where $v=\sum_{j=n+1}^{\ell} \lambda_{j} \frac{\partial}{\partial t_{j}}$. Then by (2.4), we can write $\left(\xi^{\mathrm{e}}(\mathrm{v})\right)_{\sigma}=\sum_{i=1}^{\mathrm{n}} \mu_{i} y_{\sigma i} \frac{\partial \mathrm{h}}{\partial y_{\sigma i}}$ for some complex $\mu_{1}, \ldots \mu_{n}$. This implies that

$$
\sum_{k=1}^{n}\left(\sum_{i=1}^{n} \mu_{i} a_{k i}\right) y_{\sigma}^{A}+\sum_{k=n+1}^{\ell}\left(\lambda_{k}+\sum_{i=1}^{n} \mu_{i} a_{k i}\right) y_{\sigma}^{A_{k}} \equiv 0
$$

modulo $h\left(\mathbf{y}_{\sigma}, \mathbf{t}\right)$. This implies that $\lambda_{k}=0$ for $k=n+1, \ldots$, $\ell$ and $\mu_{i}=0$ for $i=1, \ldots, n$, because the left side has no constant term. This completes the proof. It seems that the assumption in Theorem (6.8) is satisfied in many cases if $w$ is not projective space $\mathrm{P}^{\mathrm{n}}$. The following is an example where the Kodaira-spencer map is not injective.

Example (6.9). (Hashimoto- Oka[4]) Let $M$ be the algebraic surface which is the compactification of $y_{1}+y_{1}^{9} y_{2}^{16}+y_{1}^{3} y_{3}^{4}+1=0$. Then $M$ has the following invariants: $K^{2}=0, p_{g}=1$ and $\pi_{1}(M)=Z / 2 Z$. M has 27 dimensional 
effective deformation and $\operatorname{dim} H^{1}\left(M, \Theta_{W} \mid M\right)=20$. On the other hand, $H^{0}\left(M, \theta_{W} \mid M\right)=12$ and the dimension of the image of effective deformation is 18 .

\section{$\S 7$. Deformation of a Godeaux surface.}

In this section, we study the case of $n=3$. Recall that $\Xi=\Delta(P)$ is spun by $B_{0}, \ldots, B_{3}$. Let $\Xi_{i}$ be the 2-face of $\Xi$ with $B_{i} \notin \Xi_{i}$ for $i=0, \ldots, 3$. Let $P_{0}, \ldots, P_{3}$ be the vertices of $\Sigma^{*}$ which are adjacent to $P$ such that $\Delta\left(P_{i}\right)=\Xi_{i}$. We define divisors $\hat{C}_{i}$ of $w$ by $\hat{E}(P) \cap \hat{E}\left(P_{i}\right)$ and divisors $C_{i}$ of $M$ by $E(P) \cap E\left(P_{i}\right)$ for $i=0, \ldots, 3$. Let $\sigma$ be as in $\S 4$ and we denote $y_{\sigma i}$ by $y_{i}$ for simplicity. Let $A=C\left[y_{1}, y_{1}^{-}\right.$ $\left.1, \ldots, y_{3}, y_{3}^{-1}\right]$. For a polynomial $g(y)$ of $A$, we define an integer ord $\hat{\mathrm{C}}_{i} g(y)$ by the order of the zeros (or poles) of $g(y)$ along the divisor $\hat{C}_{i}$. Similarly we define ord $\mathrm{C}_{i} g(y)$ by the order of the zeros of $\left(g \mid M_{t}\right)$ along $C_{i}$. In general, we have the inequality ord $\hat{\mathrm{C}}_{i} g \leqq$ ord $_{\mathrm{C}_{i}}$.

Definition (7.1). We say that $g(y)$ has a reqular $\underline{\text { form }}$ on $C_{i}$ if ord $\hat{C}_{i} g(y)=\operatorname{ord}_{C_{i}} g(y)$.

We $f i x$ an index a for $0 \leqq a \leqq 3$. Let $\tau=\left(P, Q_{1}(\tau), Q_{2}(\tau), Q_{3}(\tau)\right)$ be a simplex of $S$ such that $Q_{1}(\tau)=P_{a}$ and let $\sigma^{-1} \cdot \tau=\left(\lambda_{i j}\right)$. Then by the definition, we have ord $\hat{C}_{a} y^{\nu}=\sum_{j=1}^{3} \nu_{j} \lambda_{j 1}$. We define $h^{a}(y, t)=\Sigma^{\prime} t_{j} y^{A_{j}}$ 
where the sum is taken for $j$ such that $B_{j} \in \Xi_{a}$. Note that $h^{a}(y, t)$ is homogeneous with respect to the weight $\left(\lambda_{11}, \lambda_{21}, \lambda_{31}\right)$ and

$$
\operatorname{ord}_{\hat{C}_{a}} y^{A}>\operatorname{ord}_{\hat{C}_{a}} h^{a}
$$

Note also that $\mathrm{h}^{\mathrm{a}}$ is irreducible in $\mathrm{A}$, because $\mathrm{C}_{\mathrm{a}}$ is an irreducible curve and the defining polynomial of $\mathrm{C}_{\mathrm{a}}$ is $\mathrm{h}^{\mathrm{a}}$ up to the multiplication of a monomial. Take $g \in A$. Let $k={ }_{\hat{C}_{i}} g$ and let $g_{k}$ be the leading term of $g$ with respect to the above weight. Then we have

Lemma (7.3). $g$ has a reqular form on $\mathrm{C}_{\mathrm{a}}$ if and only if $g_{k}$ is not zero modulo $\mathrm{h}^{\mathrm{a}}$.

Proof. We can write $g_{k}\left(y\left(y_{\tau}\right)\right)=y_{\tau 1}^{k} g^{\prime}\left(y_{\tau 2}, y_{\tau 3}\right)$. As $g\left(y\left(y_{\tau}\right)\right) \equiv g_{k}\left(y\left(y_{\tau}\right)\right)$ modulo $\left(y_{\tau 1}^{k+1}\right)$, it is easy to see that $g^{\prime} \mid C_{a} \equiv 0$ iff $g_{k} \equiv 0$ modulo $h^{a}$.

Now let $x=\sum_{j=1}^{3} x_{j} \frac{\tilde{\partial}}{\partial y_{j}}$ be a rational vector field on $w$ such that $x_{j} \in A$. We define ord $\widehat{C}_{i} x=\underset{1 \leqq j \leqq 3}{\operatorname{minimum}} \underset{\widehat{C}_{i}}{\operatorname{ord}} x_{j}$ and $\operatorname{ord}_{C_{i}} x=\underset{1 \leqq j \leqq 3}{\operatorname{minimum}} \operatorname{ord} C_{i} x_{j}$. Let $x=\sum_{j=1}^{3} x_{\tau j} \frac{\tilde{\partial}}{\partial y_{\tau j}}$ on $c_{\tau}^{3}$. Then

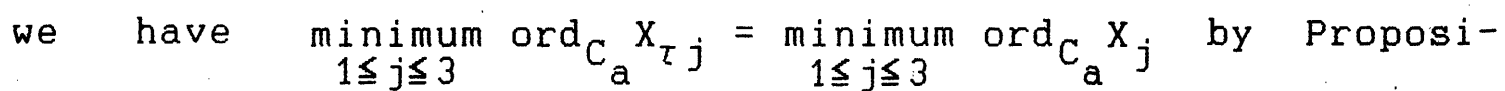
tion (6.1). In particular, $\underline{\text { if }} \underline{\mathrm{X}} \underline{\text { is }} \underline{\text { an }}$ element $\underline{\text { of }}$ $H^{0}\left(M_{t}, \theta_{W} \mid M_{t}\right)$, we have ord $C_{i} X \geq-1$ for each $\underline{i}$. Similarly let $\omega=\sum_{j=1}^{3} Y_{j} \tilde{d} y_{j}$ be a rational 1 -form such that $Y_{j} \in A$. We 
define ord ${ }_{\mathrm{C}_{i}} \omega$ and ord $\mathrm{C}_{i} \omega$ in the same way. Then we have

Lemma (7.4). (i) Let $\underline{x}$ be as above and assume that $\left\{x_{j}\right.$ \}$\quad(j=1,2,3)$ have reqular forms on $C_{a}$ and assume that $\operatorname{ord}_{\mathrm{C}} \mathrm{X} \leqq-2$ for some a. Then $x$ is not a holomorphic section of $\Theta_{W}$ over $M_{t}$.

(ii) Let $D=\sum_{i=0}^{3} n_{i} C_{i}+D^{\prime}$ be a divisor on $M_{t}$ such that the support of $D^{\prime}$ does not include any of $C_{i}(i=0, \ldots, 3)$. Let $\omega$ be as above. Assume that $\left\{Y_{j}\right\} \quad(j=1,2,3)$ have reqular forms on $C_{a}$ for some a. If ord $C_{a} \omega \leqq-n_{a}$, the restriction of $\omega$ to $M_{t}$ is not contained in $H^{0}\left(M_{t}, \Omega_{W}^{1} \mid M_{t}(D)\right)$.

For the rest of the section, we consider the following example. Let

$$
f_{\Delta}(z)=z_{0}^{2} z_{1} z_{2}^{4}+z_{1}^{2} z_{2} z_{3}^{4}+z_{2}^{2} z_{3} z_{0}^{4}+z_{3}^{2} z_{0} z_{1}^{4}
$$

and let $f(z)=f_{\Delta}(z)+\sum_{i=0}^{3} z_{i}^{11}$. Let $P=t(1,1,1,1)$. As $\Gamma^{*}(f)$ is invariant under the canonical $\mathrm{Z} / 4 \mathrm{Z}$-action, we can take $\Sigma^{*}$ to be $\mathrm{Z} / 4 \mathrm{Z}$-invariant and $\Sigma^{*}$ is canonical in the

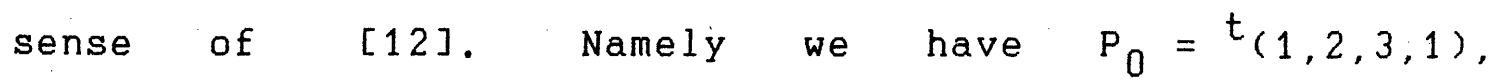

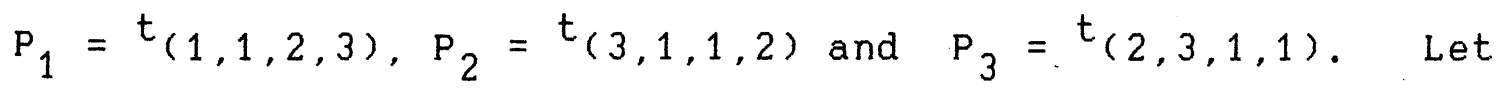
$\sigma=\left(P_{0}, P_{0}, P_{1}, R\right) \quad$ where = $\left(P_{2}+2 P_{0}+3 P_{1}+2 P\right) / 5=t(2,2,3,3)$. Let $M=E(P)$. The defining equation of $M$ in $C_{\sigma}^{3}$ is

$$
h(y)=y_{1}^{5} y_{3}^{2}+y_{2}^{5} y_{3}^{3}+y_{3}+1=0
$$


We have shown in Example, (9.11) of [12] that $\pi_{1}(M)=z / 5 z$ and $q=p_{g}=0$. This surface is known as a Godeaux surface. As $l$ is 11, the dimension of the embedded deformation is 8 . The corresponding embedded monomials are: $y_{2} y_{3}, y_{2}^{3} y_{3}^{2}, y_{1} y_{3}$, $y_{1} y_{2} y_{3}, y_{1} y_{2}^{2} y_{3}^{2}, y_{1}^{2} y_{3}, y_{1}^{2} y_{2}^{2} y_{3}^{2}$ and $y_{1}^{3} y_{2} y_{3}^{2}$. See [11]. Let $\mathrm{h}(\mathbf{y}, \mathbf{t})$ be as before. As numerical data, we have $\mathrm{K} \sim 2 \mathrm{C}_{3}-$ $\mathrm{C}_{2} \sim 2 \mathrm{C}_{1}-\mathrm{C}_{0}$ and $\mathrm{C}_{\mathrm{i}}^{2}=1$ and $\mathrm{K}^{2}=1$. Here $\mathrm{K}$ is a canonical divisor. By the Riemann-Roch theorem, we have $\chi\left(\theta_{t}\right)=-8$. We will show that

Theorem (7.5). We have $H^{0}\left(M_{t}, \theta_{t}\right)=H^{2}\left(M_{t}, \theta_{t}\right)=0$, $H^{1}\left(M_{t}, \theta_{t}\right) \cong C^{8}$ and the Kodaira- Spencer map

$$
\delta \cdot \xi^{e}: T_{t} U^{e} \rightarrow H^{1}\left(M_{t}, \theta_{t}\right)
$$

is an isomorphism.

Compare with the construction of the moduli space of the Godeaux surfaces by Miyaoka [8]. Note that $\mathbf{z} / 4 \mathbf{z}$ acts canonically on $U^{e}$ so that $M_{t} \cong M_{g t}$ for $g \in Z / 4 Z$.

Lemma (7.6). $H^{0}\left(M_{t}, \Theta_{W} \mid M_{t}\right) \cong c^{3}$ and $H^{2}\left(M_{t},{ }^{\theta_{W}} \mid M_{t}\right)=0$

Proof. Let $\tau=\left(P, P_{2}, P_{3}, R^{\prime}\right)$ where $R^{\prime}=t(3,3,2,2)$. We denote $y_{\tau i}$ by $u_{i}$ for simplicity. Then we have $y_{1}=u_{1}^{-2} u_{2}$, $y_{2}=u_{1}^{-3} u_{2}^{2}$ and $y_{3}=u_{1}^{5} u_{2}^{-5} u_{3}^{-1}$. Let $x \in H^{0}\left(M_{t}, \Theta_{W} \mid M_{t}\right)$. By the GAGA-principle, $X$ can be expressed in $\mathrm{C}_{\sigma}^{3} \cap \mathrm{M}_{\mathbf{t}}$ as $\sum_{j=1}^{3} x_{j} \frac{\tilde{\partial}}{\partial y_{j}}$ where $x_{j} \in A$.

Assertion. We can assume that $x_{j}$ has a reqular form 
on $\mathrm{C}_{2}$ and $\mathrm{C}_{3}$ simultaneously.

Proof. We may first assume that ord $\hat{C}_{3} x_{i}=\operatorname{ord}_{C_{3}} x_{i}$, using the irreducibility of $\mathrm{h}^{3}$ in $\mathrm{A}$. Assume that $\mathrm{x}_{i}$ has not a regular form on $c_{2}$. We substitute $h^{2}(y) y^{\nu}$ by $\left(h(y, t)-h^{2}(y, t)\right) y^{\nu}$ to change $x_{i}$ in a regular form on $c_{2}$ in a finite steps. Note that this operation does not decrease ord $\vec{C}_{3} x_{i}$. Thus if we change $x_{i}$ in a regular form $x^{\prime}{ }_{i}$ on $C_{2}$, we have

$$
\operatorname{ord}_{C_{3}} X_{i}=\operatorname{ord}_{C_{3}} X^{\prime}{ }_{i} \geq \operatorname{ord}_{\hat{C}_{3}} X^{\prime}{ }_{i} \geq \text { ord } \hat{C}_{3} X_{i} \text {. }
$$

This implies that ord ${ }_{\mathrm{C}_{3}} \mathrm{X}^{\prime}{ }_{i}=\operatorname{ord}_{\mathrm{C}_{3}} \mathrm{X}^{\prime}{ }_{i}$ by the regularity assumption on $C_{3}$. Assume that the monomial $y^{\nu}$ has a nonzero coefficient in $X_{i}$. As we have

$$
y^{\nu}=u_{1}^{-2 v_{1}-3 v_{2}+5 v_{3}} u_{2}^{\nu_{1}+2 v_{2}-5 v_{3}} u_{3}^{-\nu_{3}} \text {, }
$$

we must have $v_{1}+2 v_{2}+1 \geqq 5 v_{3} \geq 2 v_{1}+3 v_{2}-1$. Combine this with $\nu_{1} \geqq-\delta_{i 1}, \quad \nu_{2} \geq-\delta_{i 2}$ where $\delta_{i j}$ is the Kronecker's symbol. The possible cases are $y_{2}^{2} y_{3} \frac{\tilde{\partial}}{\partial y_{i}}(i=1,2,3), y_{1}^{2} y_{2}^{-}$ $1 \frac{\tilde{\partial}}{\partial y_{2}}, y_{1} y_{2}^{-1} \frac{\tilde{\partial}}{\partial y_{2}}, y_{1}^{-1}, y_{2} \frac{\tilde{\partial}}{\partial y_{1}}$ and $\frac{\tilde{\partial}}{\partial y_{i}}$. After checking their linear combinations in detail, we conclude that $H^{0}\left(M_{t}, \theta_{W} \mid M_{t}\right)=\operatorname{Can}\left(M_{t}, \theta_{W}\right)$.

Now we consider $H^{2}\left(M_{t}, \theta_{W} \mid M_{t}\right)$. By the serre duality, this is isormophic to $H^{0}\left(M_{t}, \Omega_{W}^{1}(K)\right) \cong H^{0}\left(M_{t}, \Omega_{W}^{1} \mid M_{t}\left(2 C_{1}-C_{0}\right)\right)$ 
where $\Omega_{\mathrm{W}}^{1}$ is the sheaf of the germs of 1 -forms on $w$. Let $\omega=\sum_{i=1}^{3} Y_{i} \tilde{d} y_{i}$ be a rational 1-form with $Y_{i} \in A$ and assume that the restriction of $\omega$ is in $H^{0}\left(M_{t}, \Omega_{W}^{1} \mid M_{t}\left(2 C_{1}-C_{0}\right)\right)$. Let $\mathbf{y}^{\nu}$ be a monomial with a non-zero coefficient in $Y_{i}$. Then by Lemma (7.4), we have $\nu_{1} \geqq-2+\delta_{i 1}, \nu_{2} \geqq 1+\delta_{i 2}$ and $v_{1}+2 v_{2} \geqq 5 v_{3} \geqq 2 v_{1}+3 v_{2}$. This has no integral solution. This implies that $\mathrm{H}^{2}\left(M_{\mathbf{t}}, \theta_{\mathbf{W}} \mid \mathrm{M}_{\mathbf{t}}\right)=0$, completing the proof of Lemma (7.6).

Proof of Theorem (7.5). We consider the exact sequence (1.4). Considering the section $\varphi$ of $H^{0}\left(M_{t}, \nu_{t}\right)$ such that $\varphi_{\sigma}=1$, we see that $\mathrm{N}_{\mathbf{t}}=\left[5 \mathrm{C}_{3}\right]$. Thus by Riemann-Roch theorem, we have $\chi\left(\nu_{t}\right)=11, \chi\left(\theta_{t}\right)=-8$ and $\chi\left(\Theta_{W} \mid M_{t}\right)=3$. This implies that $H^{1}\left(M_{t}, \Theta_{W} \mid M_{t}\right)=H^{2}\left(M_{t}, \nu_{t}\right)=0$ and $H^{2}\left(M_{t}, \theta_{t}\right)=H^{0}\left(M_{t}, \theta_{t}\right)=0$ and $H^{1}\left(M_{t}, \theta_{t}\right) \cong c^{8}$. This completes the proof by Theorem (6.8).

\section{References}

[1] V.I. Danilov, The geometry of toric varieties, Russian Math. Surveys, 33:2 (1978), 97-154.

[2] M. Demazure, Sous-groupes algébriques de rang maximum du groupe de Cremona, Ann. Sci. Ecole Norm. Sup., (4) 3 (1970), 507-588. 
[3] F. Ehlers, Eine Klasse komplexer Mannigfaltigkeiten und die Auflösung einer isolierter Singularitäten, Math. Ann., 218 (1975), 127-156.

[4] N. Hashimoto and M. Oka, Example of an algebraic surface whose effective deformation is not injective, in preparation.

[5] G. Kempf, F. Knudsen, D. Mumford, and B. Saint-Donat, Toroidal Embeddings, 339, Springer, Berlin-HeidelbergNew York, 1973.

[6] K. Kodaira and D.C. Spencer, on deformations of complex structures I, II, Annals of Math., 67 (1958), 328-466.

[7] K. Kodaira, Complex Manifolds and Deformation of Complex Structures, Springer, Berlin-Heiderberg-New York, 1985.

[8] Y. Miyaoka, Tricanonical Maps of Numerical Godeaux Surfaces, Inventiones Math., 34 (1976), 99-111.

[9] T. Oda, Lectures on torus embeddings and applications, 58, Springer-Verlag, Berlin-Heiderberg-New York, 1978.

[10] T. Oda, Convex body and algebraic geometry (in Japanese), Kinokuniya, Tokyo, 1985.

[11] M. Oka, Examples of Algebraic Surfaces with $q=0$ and $p_{g} \leqq 1$ which are Locally Hypersurfaces, preprint. 
[12] M. Oka, On the Resolution of Hypersurface Singularities, Advanced study in Pure Mathematics, 8 (1986), $405-436$.

[13] J.P. Serre, Géométrie Algébrique et Géométrie Analytique, Ann. Innst. Fourier, 36 (1956), 1-42. 